\title{
On magnetohydrodynamic turbulence and angular momentum transport in accretion disk boundary layers
}

\author{
Chi-kwan Chan ${ }^{1}$ and Martin E. Pessah ${ }^{2}$ \\ ${ }^{1}$ NORDITA, Roslagstullsbacken 23, 10691 Stockholm, Sweden \\ email: ckch@nordita.org \\ ${ }^{2}$ Niels Bohr International Academy, Niels Bohr Institute \\ Blegdamsvej 17, 2100 Copenhagen Ø, Denmark \\ email: mpessah@nbi.dk
}

\begin{abstract}
The physical modeling of the accretion disk boundary layer, the region where the disk meets the surface of the accreting star, usually relies on the assumption that angular momentum transport is opposite to the radial angular frequency gradient of the disk. The standard model for turbulent shear viscosity, widely adopted in astrophysics, satisfies this assumption by construction. However, this behavior is not supported by numerical simulations of turbulent magnetohydrodynamic (MHD) accretion disks, which show that angular momentum transport driven by the magnetorotational instability is inefficient in this inner disk region. I will discuss the results of a recent study on the generation of hydromagnetic stresses and energy density in the boundary layer around a weakly magnetized star. Our findings suggest that although magnetic energy density can be significantly amplified in this region, angular momentum transport is rather inefficient. This seems consistent with the results obtained in numerical simulations and suggests that the detailed structure of turbulent MHD boundary layers could differ appreciably from those derived within the standard framework of turbulent shear viscosity.
\end{abstract}

Keywords. accretion disks, MHD, turbulence

\section{Introduction}

For an accretion disk to merge smoothly to the surface of the central accreting star, its angular velocity $\Omega(r)$ must decrease inwards in the inner disk to match the subKeplerian stellar spin rate $\Omega_{\star}$ (Frank et al. 2002; Hartmann 2009; Armitage 2010). It is easy to derive from the standard accretion disk model that half of the accretion energy is released in this so called accretion disk boundary layer (ADBL). Understanding the detailed physics within this thin layer, therefore, is of great astrophysical interest (Piro \& Bildsten 2004; Balsara et al. 2009; Inogamov \& Sunyaev 2010).

According to standard models, the structure of ADBL depends entirely on turbulent angular momentum transport. On the one hand, standard models implicitly assume that the turbulent stress is linearly proportional to the local shear (Shakura \& Sunyaev 1973). On the other hand, it is widely accepted that the magnetorotational instability (MRI) is responsible to drive turbulence in the accretion disk (Balbus \& Hawley 1991, 1998). Given that the shear rate, $q \equiv-d \ln \Omega / d \ln r$, is negative in the boundary layer, the MRI is inactive and the standard ADBL model breaks down.

To understand the behavior of the ADBL and derive a self-consistent model, we study the local evolution of magnetohydrodynamic (MHD) waves when they are stable to the MRI. We explain how shearing MHD waves can generate strong toroidal magnetic fields without leading to efficient angular momentum transport. 


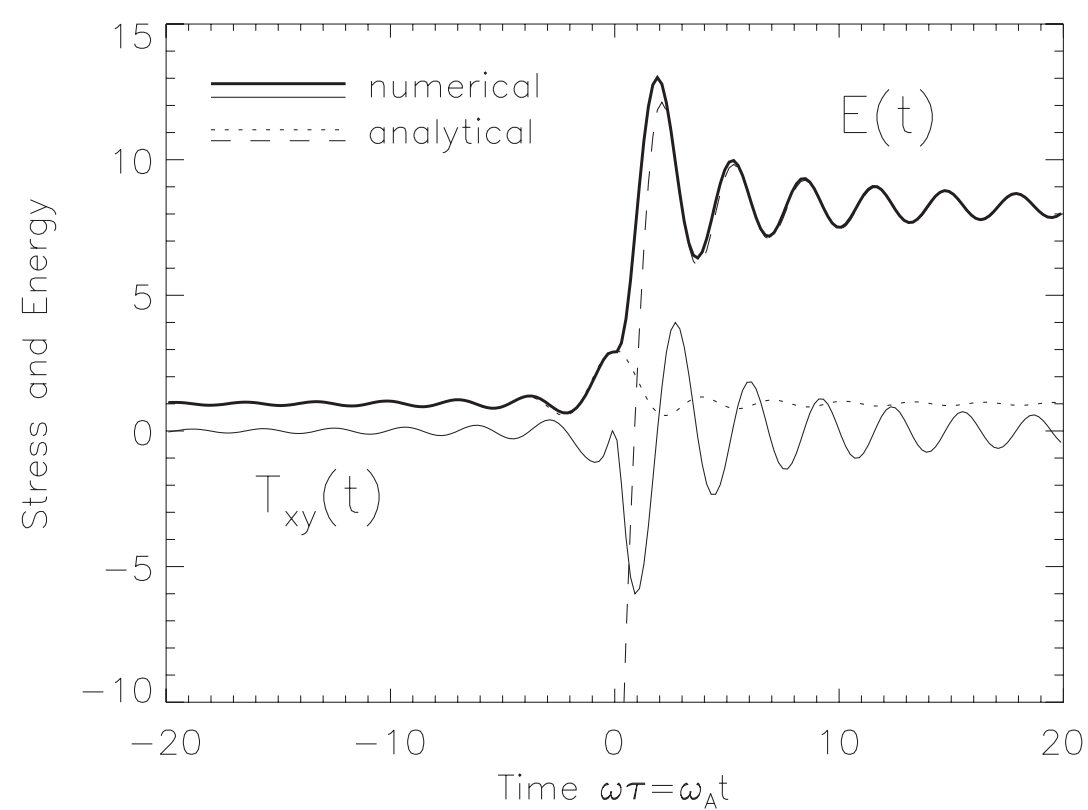

Figure 1. The thick and thin solid lines correspond, respectively, to the numerical total energy $E(t)$ and total stress $T_{x y}(t)$. The dotted and dashed lines show the analytic approximation for the energy, equation (2.4).

\section{Local Model for MHD Accretion Disk Boundary Layers}

We employ the standard shearing box approximation (Hawley et al. 1995) and follow the notation in Pessah \& Chan (2012) to denote the velocity and magnetic fluctuations by $\boldsymbol{u}$ and $\boldsymbol{b}$, respectively. By assuming the flow is incompressible and considering only a single (shearing) Fourier mode at wavenumber $\boldsymbol{k} \equiv\left(k_{x}, k_{y}, k_{z}\right)$, all the non-linear terms in the ideal MHD equations vanish identically (Goodman \& Xu 1994).

To isolate the interesting non-MRI dynamics, we focus only on $z$-independent perturbation. That is, we set $k_{z} \equiv 0$. The non-trivial MHD can be summarized in the following second-order ordinary differential equation (see Pessah \& Chan 2012, for derivation):

$$
\frac{d \hat{b}_{x}}{d \tau^{2}}+\Gamma(\tau) \frac{d \hat{b}_{x}}{d \tau}+\omega^{2} \hat{b}_{x}=0
$$

In the above equation, the independent variable $\tau \equiv k_{x}(t) / k_{y}=q \Omega_{0} t$ is the dimensionless time, where $\Omega_{0}$ is the corotating frequency of the shearing box; the dependent variable $\hat{b}_{x}$ is the Fourier coefficient of $b_{x}$ at $\boldsymbol{k}$. The symbol $\omega \equiv \omega_{\mathrm{A}} / q \Omega_{0}$ is the dimensionless form of the (constant) Alfvén frequency $\omega_{\mathrm{A}} \equiv \boldsymbol{B}_{0} \cdot \boldsymbol{k}$, where $\boldsymbol{B}_{0}$ is the background magnetic field. Finally, we use the shorthand $\Gamma(\tau) \equiv 2 \tau /\left(\tau^{2}+1\right)$. Other components of the fluctuations can be easily derive from $\hat{b}_{x}$ by

$$
\hat{u}_{x}=-\frac{i}{\omega} \frac{d \hat{b}_{x}}{d \tau}
$$

and the divergence-less conditions $\nabla \cdot \boldsymbol{u}=\nabla \cdot \boldsymbol{b}=0$.

Equation (2.1) does not have an analytic solution. Nevertheless, it reduces to a Bessel equation in the limit $\tau \rightarrow \pm \infty$. By keeping up to the first order terms in $1 / \omega \tau$, we can derive the (mean) total stress $T_{x y} \equiv\left\langle u_{x} u_{y}-b_{x} b_{y}\right\rangle$ and the (mean) energy density 


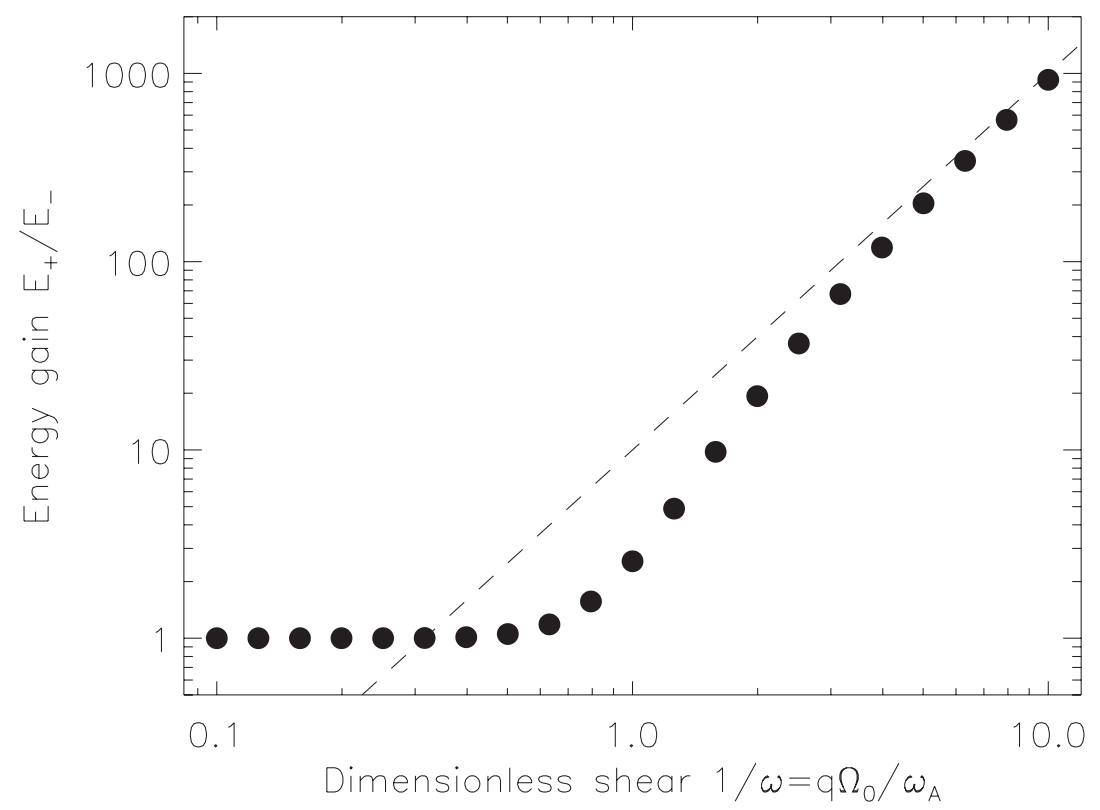

Figure 2. The filled circles represent the value of the amplification factor $E_{+} / E_{-}$for different values of the shear. The dashed line shows the function $10 / \omega^{2} \equiv 10\left(q \Omega_{0} / \omega_{\mathrm{A}}\right)^{2}$, which is in good agreement with the numerical results for strong shear. This asymptotic behavior is insensitive of the initial conditions as long as $C_{-} \neq 0$. See Pessah \& Chan (2012) for details.

$E \equiv\left\langle u^{2}+b^{2}\right\rangle / 2$, where $\langle\cdot\rangle$ stands for the spatial average:

$$
\begin{aligned}
T_{x y} & \approx-\frac{2}{\omega^{2} \tau}\left[\left(|S|^{2}-|C|^{2}\right) \cos (2 \omega \tau)+\left(S^{*} C+S C^{*}\right) \sin (2 \omega \tau)\right], \\
E & \approx-\frac{1}{\omega^{3} \tau}\left[\left(|S|^{2}-|C|^{2}\right) \sin (2 \omega \tau)-\left(S^{*} C+S C^{*}\right) \cos (2 \omega \tau)\right]+\frac{|S|^{2}-|C|^{2}}{\omega^{2}} .
\end{aligned}
$$

The complex integration constants $S$ and $C$ are determined by the initial conditions; and the star symbol denotes complex conjugation. Note that the energy balance equation $d E / d t=q \Omega_{0} T_{x y}$ is satisfied up to first order.

We plot the numerical solutions for the energy $E(t)$ and the stress $T_{x y}(t)$ in Figure 1 by thick and thin solid lines, respectively. The dotted and dashed lines show our analytic approximation with two different sets of integration constants. Note that the energy increases significantly around $\tau=0$ although the stress keeps oscillating around zero. The physical meaning is that the shearing MHD waves can extract energy from the background without increasing the time-averaged stress. Hence, the dissipation of magnetic energy in the ADBL is inefficient.

Let $E_{ \pm} \equiv \lim _{\tau \rightarrow \pm \infty} E(\tau)$. Despite the fact that we cannot analytically solve for the amplification factor $E_{+} / E_{-}$, we can still draw important conclusions from the properties of the numerical solutions. We plot the amplification factor as a function of $1 / \omega$ in Figure 2. In the limit of weak shear, the pure Alfvén waves cannot lead to net energy gain. Nevertheless, for strong shear $q \gg \omega_{\mathrm{A}} / \Omega_{0}$, or $1 / \omega \gg 1$, the amplification factor follows a power law,

$$
\frac{E_{+}}{E_{-}} \approx \frac{10}{\omega^{2}}=10\left(\frac{q \Omega_{0}}{\omega_{\mathrm{A}}}\right)^{2} .
$$

This is remarkable because the amplification is unbounded without a linear instability. 


\section{Conclusions}

Understanding the relationship between the stress and the shear rate is essential in modeling the ADBL. In this proceeding, we point out that the standard turbulent accretion disk model is inapplicable in the boundary layer because the MRI is inactive there. We provide the key steps to solve stable shearing MHD waves, which describes the physics better. Although the energy of the waves can be significantly amplified, the time-averaged stress remains zero. Our findings agree with global MHD simulations of accretion disks performed by Armitage (2002); Steinacker \& Papaloizou (2002).

Our results suggest that the ADBL will be strongly magnetized. However, the magnetic fields will not be able to transport angular momentum nor dissipate to heat up the plasma. One direct consequence is that the angular momentum transport from an accretion disk to a central accreting star, as predicted by the standard ADBL models, is suppressed. Some other physical mechanism is therefore needed to spin up weakly magnetized stars.

\section{References}

Armitage, P. J. 2002, MNRAS, 330, 895

-. 2010, Astrophysics of Planet Formation

Balbus, S. A. \& Hawley, J. F. 1991, ApJ, 376, 214

-. 1998, Reviews of Modern Physics, 70, 1

Balsara, D. S., Fisker, J. L., Godon, P., \& Sion, E. M. 2009, ApJ, 702, 1536

Frank, J., King, A., \& Raine, D. J. 2002, Accretion Power in Astrophysics: Third Edition

Goodman, J. \& Xu, G. 1994, ApJ, 432, 213

Hartmann, L. 2009, Accretion Processes in Star Formation: Second Edition (Cambridge University Press)

Hawley, J. F., Gammie, C. F., \& Balbus, S. A. 1995, ApJ, 440, 742

Inogamov, N. A. \& Sunyaev, R. A. 2010, Astronomy Letters, 36, 848

Pessah, M. E. \& Chan, C.-k. 2012, ApJ, 751, 48

Piro, A. L. \& Bildsten, L. 2004, ApJ, 610, 977

Shakura, N. I. \& Sunyaev, R. A. 1973, A\&A, 24, 337

Steinacker, A. \& Papaloizou, J. C. B. 2002, ApJ, 571, 413 